NBER WORKING PAPER SERIES

DYNAMIC ASSET ALLOCATION WITH EVENT RISK

\author{
Jun Liu \\ Francis A. Longstaff \\ Jun Pan \\ Working Paper 9103 \\ http://www.nber.org/papers/w9103 \\ NATIONAL BUREAU OF ECONOMIC RESEARCH \\ 1050 Massachusetts Avenue \\ Cambridge, MA 02138
}

August 2002

Liu and Longsta are with the Anderson School at UCLA and Pan is with the MIT Sloan School of Management. We are particularly grateful for helpful discussions with Tony Bernardo and Pedro Santa-Clara, for the comments of Jerome Detemple, Harrison Hong, Paul Pfleiderer, Raman Uppal, and participants at the 2001 Western Finance Association meetings, and for the many insightful comments and suggestions of the editor Richard Green and the referee. All errors are our responsibility. The views expressed herein are those of the authors and not necessarily those of the National Bureau of Economic Research.

(C) 2002 by Jun Liu, Francis A. Longstaff and Jun Pan. All rights reserved. Short sections of text, not to exceed two paragraphs, may be quoted without explicit permission provided that full credit, including $(\mathbb{C}$ notice, is given to the source. 
Dynamic Asset Allocation With Event Risk

Jun Liu, Francis A. Longstaff and Jun Pan

NBER Working Paper No. 9103

August 2002

JEL No. G1

\begin{abstract}
Major events often trigger abrupt changes in stock prices and volatility. We study the implications of jumps in prices and volatility on investment strategies. Using the event-risk framework of Duffie, Pan, and Singleton (2000), we provide analytical solutions to the optimal portfolio problem. Event risk dramatically affects the optimal strategy. An investor facing event risk is less willing to take leveraged or short positions. The investor acts as if some portion of his wealth may become illiquid and the optimal strategy blends both dynamic and buy-and-hold strategies. Jumps in prices and volatility both have important effects.
\end{abstract}

Jun Liu

The Anderson School at UCLA

110 Westwood Plaza

Los Angeles, CA 90095-1481

Francis A. Longstaff

The Anderson School at UCLA

110 Westwood Plaza

Los Angeles, CA 90095-1481

and NBER

francis.longstaff@anderson.ucla.edu

Jun Pan

MIT Sloan School of Management

50 Memorial Drive, E52-454

Cambridge, MA 02142 
One of the inherent hazards of investing in financial markets is the risk of a major event precipitating a sudden large shock to security prices and volatilities. There are many examples of this type of event, including, most recently, the September 11th, 2001 terrorist attacks. Other recent examples include the stock market crash of October 19, 1987 in which the Dow index fell by 508 points, the October 27, 1997 drop in the Dow index by more than 554 points, and the flight to quality in the aftermath of the Russian debt default where swap spreads increased on August 27, 1998 by more than twenty times their daily standard deviation, leading to the downfall of Long Term Capital Management and many other highly leveraged hedge funds. Each of these events was accompanied by major increases in market volatility. ${ }^{1}$

The risk of event-related jumps in security prices and volatility changes the standard dynamic portfolio choice problem in several important ways. In the standard problem, security prices are continuous and instantaneous returns have infinitesimal standard deviations; an investor considers only small local changes in security prices in selecting a portfolio. With event-related jumps, however, the investor must also consider the effects of large security price and volatility changes when selecting a dynamic portfolio strategy. Since the portfolio that is optimal for large returns need not be the same as that for small returns, this creates a strong conflict that must be resolved by the investor in selecting a portfolio strategy.

This paper studies the implications of event-related jumps in security prices and volatility on optimal dynamic portfolio strategies. In modeling event-related jumps, we use the double-jump framework of Duffie, Pan, and Singleton (2000). This framework is motivated by evidence by Bates (2000) and others of the existence of volatility jumps, and has received strong empirical support from the data. ${ }^{2}$ In this model, both the security price and the volatility of its returns follow jump-diffusion processes. Jumps are triggered by a Poisson event which has an intensity proportional to the level of volatility. This intuitive framework closely parallels the behavior of actual financial markets and allows us to study directly the effects of event risk on portfolio choice.

To make the intuition behind the results as clear as possible, we focus on the simplest case where an investor with power utility over end-of-period wealth allocates 
his portfolio between a riskless asset and a risky asset that follows the double-jump process. Because of the tractability provided by the affine structure of the model, we are able to reduce the Hamilton-Jacobi-Bellman partial differential equation for the indirect utility function to a set of ordinary differential equations. This allows us to obtain an analytical solution for the optimal portfolio weight. In the general case, the optimal portfolio weight is given by solving a simple pair of nonlinear equations. In a number of special cases, however, closed-form solutions for the optimal portfolio weight are readily obtained.

The optimal portfolio strategy in the presence of event risk has many interesting features. One immediate effect of introducing jumps into the portfolio problem is that return distributions may display more skewness and kurtosis. While this has an important influence on the portfolio chosen, the full implications of event risk for dynamic asset allocation run much deeper. We show that the threat of event-related jumps makes an investor behave as if he faced short-selling and borrowing constraints even though none are imposed. This result parallels Longstaff (2001) where investors facing illiquid or nonmarketable assets restrict their portfolio leverage. Interestingly, we find that the optimal portfolio is a blend of the optimal portfolio for a continuoustime problem and the optimal portfolio for a static buy-and-hold problem. Intuitively, this is because when an event-related jump occurs, the portfolio return is on the same order of magnitude as the return that would be obtained from a buy-andhold portfolio over some finite horizon. Since these two returns have the same effect on terminal wealth, their implications for portfolio choice are indistinguishable, and event risk can be interpreted or viewed as a form of liquidity risk. This perspective provides new insights into the effects of event risk on financial markets.

To illustrate our results, we provide two examples. In the first, we consider a model where the risky asset follows a jump-diffusion process with deterministic jump sizes, but where return volatility is constant. This special case parallels Merton (1971) who solves for the optimal portfolio weight when the riskless rate follows a jump-diffusion process. We find that an investor facing jumps may choose a portfolio very different from the portfolio that would be optimal if jumps did not occur. In general, the investor holds less of the risky asset when event-related price jumps can 
occur. This is true even when only upward price jumps can occur. Intuitively, this is because the effect of jumps on return volatility dominates the effect of the resulting positive skewness. Because event risk is constant over time in this example, the optimal portfolio does not depend on the investor's horizon.

In the second example, we consider a model where both the risky asset and its return volatility follow jump-diffusion processes with deterministic jump sizes. The stochastic volatility model studied by Liu (1999) can be viewed as a special case of this model. As in Liu, the optimal portfolio weight does not depend on the level of volatility. The optimal portfolio weight, however, does depend on the investor's horizon since the probability of an event is time varying through its dependence on the level of volatility. We find that volatility jumps can have a significant effect on the optimal portfolio above and beyond the effect of price jumps. Surprisingly, investors may even choose to hold more of the risky asset when there are volatility jumps than otherwise. Intuitively, this means that the investor can partially hedge the effects of volatility jumps on his indirect utility through the offsetting effects of price jumps. Note that this hedging behavior arises because of the static buy-andhold component of the investor's portfolio problem; this static jump-hedging behavior differs fundamentally from the usual dynamic hedging of state variables that occurs in the standard pure-diffusion portfolio choice problem.

We provide an application of the model by calibrating it to historical U.S. data and examining its implications for optimal portfolio weights. The results show that even when large jumps are very infrequent, an investor still finds it optimal to reduce his exposure to the stock market significantly. These results suggest a possible reason why historical levels of stock market participation have tended to be lower than would be optimal in many classical portfolio choice models. While volatility jumps are qualitatively important for optimal portfolio choice, the calibrated exercise shows that they generally have less impact than price jumps.

Since the original work by Merton (1971), the problem of portfolio choice in the presence of richer stochastic environments has become a topic of increasing interest. Recent examples of this literature include Brennan, Schwartz, and Lagnado (1997) on asset allocation with stochastic interest rates and predictability in stock returns, Kim 
and Omberg (1996), Campbell and Viceira (1999), Barberis (2000), and Xia (2001) on predictability in stock returns (with or without learning), Lynch (2001) on portfolio choice and equity characteristics, Schroder and Skiadas (1999) on a class of affine diffusion models with stochastic differential utility, Balduzzi and Lynch (1999) on transaction costs and stock return predictability, and Brennan and Xia (1998), Liu (1999), Wachter (1999), Campbell and Viceira (2001) on stochastic interest rates, and Ang and Bekaert (2000) on time varying correlations. Aase (1986), and Aase and Øksendal (1988) study the properties of admissible portfolio strategies in jump diffusion contexts. Aase (1984), Jeanblanc-Picqué and Pontier (1990), and Bardhan and Chao (1995) provide more general analyses of portfolio choice when asset price dynamics are discontinuous. Although Merton (1971), Common (2000), and Das and Uppal (2001) study the effects of price jumps and Liu (1999), Chacko and Viceira (2000), and Longstaff (2001) study the effects of stochastic volatility, this paper contributes to the literature by being the first to study the effects of event-related jumps in both stock prices and volatility. ${ }^{3}$

The remainder of this paper is organized as follows. Section I presents the eventrisk model. Section II provides analytical solutions to the optimal portfolio allocation problem. Section III presents the examples and provides numerical results. Section IV calibrates the model and examines the implications for optimal portfolio choice. Section V summarizes the results and make concluding remarks.

\section{The Event-Risk Model}

We assume that there are two assets in the economy. The first is a riskless asset paying a constant rate of interest $r$. The second is a risky asset whose price $S_{t}$ is subject to event-related jumps. Specifically, the price of the risky asset follows the process

$$
\begin{gathered}
d S_{t}=\left(r+\eta V_{t}-\mu \lambda V_{t}\right) S_{t} d t+\sqrt{V_{t}} S_{t} d Z_{1 t}+X_{t} S_{t-} d N_{t}, \\
d V_{t}=\left(\alpha-\beta V_{t}-\kappa \lambda V_{t}\right) d t+\sigma \sqrt{V_{t}} d Z_{2 t}+Y_{t} d N_{t}
\end{gathered}
$$

where $Z_{1}$ and $Z_{2}$ are standard Brownian motions with correlation $\rho, V$ is the in- 
stantaneous variance of diffusive returns, and $N$ is a Poisson process with stochastic arrival intensity $\lambda V$. The parameters $\alpha, \beta, \kappa, \lambda$, and $\sigma$ are all assumed to be nonnegative. The variable $X$ is a random price-jump size with mean $\mu$ and is assumed to have support on $(-1, \infty)$ which guarantees the positivity (limited liability) of $S$. Similarly, $Y$ is a random volatility-jump size with mean $\kappa$ and is assumed to have support on $[0, \infty)$ to guarantee that $V$ remains positive. In general, the jump sizes $X$ and $Y$ can be jointly distributed with nonzero correlation. The jump sizes $X$ and $Y$ are also assumed to be independent across jump times and independent of $Z_{1}, Z_{2}$, and $N$.

Given these dynamics, the price of the risky asset follows a stochastic-volatility jump-diffusion process and is driven by three sources of uncertainty: (1) diffusive price shocks from $Z_{1},(2)$ diffusive volatility shocks from $Z_{2}$, and (3) realizations of the Poisson process $N$. Since a realization of $N$ triggers jumps in both $S$ and $V$, a realization of $N$ has the natural interpretation of a financial event affecting both prices and market volatilities. In this sense, this model is ideal for studying the effects of event risk on portfolio choice. Because the jump sizes $X$ and $Y$ are random, however, it is possible for the arrival of an event to result in a large jump in $S$ and only a small jump in $V$, or a small jump in $S$ and a large jump in $V$. This feature is consistent with observed market behavior; although financial market events are generally associated with large movements in both prices and volatility, jumps in only prices or only volatility can occur. Since $\mu$ is the mean of the price-jump size $X$, the term $\mu \lambda V S$ in equation (1) compensates for the instantaneous expected return introduced by the jump component of the price dynamics. As a result, the instantaneous expected rate of return equals the riskless rate $r$ plus a risk premium $\eta V$. This form of the risk premium follows from Merton (1980) and is also used by Liu (1999), Pan (2002), and many others. Note that the risk premium compensates the investor for both the risk of diffusive shocks and the risk of jumps. ${ }^{4}$

These dynamics also imply that the instantaneous variance $V$ follows a meanreverting square-root jump-diffusion process. The Heston (1993) stochastic-volatility model can be obtained as a special case of this model by imposing the condition that $\lambda=0$, which implies that jumps do not occur. Liu (1999) provides closed-form 
solutions to the portfolio problem for this special case. ${ }^{5}$ Also nested as special cases are the stochastic-volatility jump-diffusion models of Bates (2000) and Bakshi, Cao, and Chen (1997). Again, since $\kappa$ is the mean of the volatility jump size $Y, \kappa \lambda V$ in the drift of the process for $V$ compensates for the jump component in volatility.

This bivariate jump-diffusion model is an extended version of the double-jump model introduced by Duffie, Pan, and Singleton (2000). Note that this model falls within the affine class because of the linearity of the drift vector, diffusion matrix, and intensity process in the state variable $V$. The double-jump framework has received a significant amount of empirical support because of the tendency for both stock prices and volatility to exhibit jumps. For example, a recent paper by Eraker, Johannes, and Polson (2000) finds strong evidence of jumps in volatility even after accounting for jumps in stock returns. ${ }^{6}$ Duffie, Pan, and Singleton also show that the doublejump model implies volatility "smiles" or skews for stock options that closely match the volatility skews observed in options markets. ${ }^{7}$

\section{Optimal Dynamic Asset Allocation}

In this section, we focus on the asset allocation problem of an investor with power utility

$$
U(x)= \begin{cases}\frac{1}{1-\gamma} x^{1-\gamma}, & \text { if } x>0 \\ -\infty, & \text { if } x \leq 0\end{cases}
$$

where $\gamma>0$, and the second part of the utility specification effectively imposes a non-negative wealth constraint. This constraint is consistent with Dybvig and Huang (1988) who show that requiring wealth to be non-negative rules out arbitrages of the type described by Harrison and Kreps (1979). As demonstrated by Kraus and Litzenberger (1976), an investor with this utility function has a preference for positive skewness.

Given the opportunity to invest in the riskless and risky assets, the investor starts with a positive initial wealth $W_{0}$ and chooses, at each time $t, 0 \leq t \leq T$, to invest a fraction $\phi_{t}$ of his wealth in the risky asset so as to maximize the expected 
utility of his terminal wealth $W_{T}$,

$$
\max _{\left\{\phi_{t}, 0 \leq t \leq T\right\}} E_{0}\left[U\left(W_{T}\right)\right]
$$

where the wealth process satisfies the self-financing condition

$$
d W_{t}=\left(r+\phi_{t}(\eta-\mu \lambda) V_{t}\right) W_{t} d t+\phi_{t} \sqrt{V_{t}} W_{t} d Z_{1 t}+X_{t} \phi_{t-} W_{t-} d N_{t}
$$

Although the model could be extended to allow for intermediate consumption, we use this simpler specification to focus more directly on the intuition behind the results.

Before solving for the optimal portfolio strategy, let us first consider how jumps affect the nature of the returns available to an investor who invests in the risky asset. When a risky asset follows a pure diffusion process without jumps, the variance of returns over an infinitesimal time period $\Delta t$ is proportional to $\Delta t$. This implies that as $\Delta t$ goes to zero, the uncertainty associated with the investor's change in wealth $\Delta W$ also goes to zero. Thus, the investor can rebalance his portfolio after every infinitesimal change in his wealth. Because of this, the investor retains complete control over his portfolio composition; his actual portfolio weight is continuously equal to the optimal portfolio weight. An important implication of this is that an investor with leveraged or short positions in a market with continuous prices can always rebalance his portfolio quickly enough to avoid negative wealth if the market turns against him.

The situation is very different, however, when asset price paths are discontinuous because of event-related jumps. For example, given the arrival of a jump event at time $t$, the uncertainty associated with the investor's change in wealth $\Delta W_{t}=W_{t}-W_{t-}$ does not go to zero. Thus, when a jump occurs, the investor's wealth can change significantly from its current value before the investor has a chance to rebalance his portfolio. An immediate implication of this is that the investor's portfolio weight is not completely under his control at all times. For example, the actual portfolio weight will typically differ from the optimal portfolio weight immediately after a jump occurs. This implies that significant amounts of portfolio rebalancing may be 
observed in markets after an event-related jump occurs. Without complete control over his portfolio weight, however, an investor with large leveraged or short positions may not be able to rebalance his portfolio quickly enough to avoid negative wealth.

Because of this, the investor not only faces the usual local-return risk that appears in the standard pure diffusion portfolio selection problem, but also the risk that large changes in his wealth may occur before having the opportunity to adjust his portfolio. This latter risk is essentially the same risk faced by an investor who holds illiquid assets in his portfolio; an investor holding illiquid assets may also experience large changes before having the opportunity to rebalance his portfolio. Because of this event-related "illiquidity" risk, the only way that the investor can guarantee that his wealth remains positive is by avoiding portfolio positions that are one jump away from ruin. This intuition is summarized in the following proposition which places bounds on admissible portfolio weights.

PROPOSITION 1: Bounds on Portfolio Weights. Suppose that for any $t, 0<t \leq T$, we have

$$
0<E_{t}\left[\exp \left(-\int_{t}^{T} \lambda V_{s} d s\right)\right]<1,
$$

where $\lambda V_{t}$ is the jump arrival intensity. Then, at any time $t$, the optimal portfolio weight $\phi_{t}^{*}$ for the asset allocation problem must satisfy

$$
1+\phi_{t}^{*} X_{\mathrm{Inf}}>0 \quad \text { and } \quad 1+\phi_{t}^{*} X_{\mathrm{Sup}}>0
$$

where $X_{\mathrm{Inf}}$ and $X_{\mathrm{Sup}}$ are the lower and upper bounds of the support of $X_{t}$ (the random price jump size). In particular, if $X_{\mathrm{Inf}}<0$ and $X_{\text {Sup }}>0$,

$$
-\frac{1}{X_{\text {Sup }}}<\phi_{t}^{*}<-\frac{1}{X_{\text {Inf }}} .
$$

Proof: See Appendix.

Thus, the investor restricts the amount of leverage or short selling in his portfolio as a hedge against his inability to continuously control his portfolio weight. If the random price jump size $X$ can take any value on $(-1, \infty)$, then this proposition 
implies that the investor will never take a leveraged or short position in the risky asset.

These results parallel Longstaff (2001) who studies dynamic asset allocation in a market where the investor is restricted to trading strategies that are of bounded variation. In his model, the investor protects himself against the risk of not being able to trade his way out of a leveraged position quickly enough to avoid negative wealth by restricting his portfolio weight to be between zero and one. Intuitively, the reason for this is the same as in our model. Having to hold a portfolio over a jump event has essentially the same effect on terminal wealth as having a buy-and-hold portfolio over some discrete horizon. In this sense, the problem of illiquidity parallels that of event-related jumps. Interestingly, discussions of major financial market events in the financial press often link the two problems together.

One issue that is not formally investigated in this paper is the role of options in alleviating the cost associated with the jump risk. Intuitively, put options could be used to hedge against the negative jump risk, allowing investors to break the jump-induced constraint and hold leveraged positions in the underlying risky asset. ${ }^{8}$ In practice, the benefit of such option strategies depends largely on the cost of such insurance against the jump risk. Moreover, in a dynamic setting with jump risk, it might be hard to perfectly hedge the jump risk with finitely many options. Putting these complications aside, it is potentially fruitful to introduce options to the portfolio problem, particularly in light of our results on the jump-induced constraints. ${ }^{9} \mathrm{~A}$ formal treatment, however, is beyond the scope of this paper.

We now turn to the asset allocation problem in equations (4) and (5). In solving for the optimal portfolio strategy, we adopt the standard stochastic control approach. Following Merton (1971), we define the indirect utility function by

$$
J(W, V, t)=\max _{\left\{\phi_{s}, t \leq s \leq T\right\}} E_{t}\left[U\left(W_{T}\right)\right] .
$$

The principle of optimal stochastic control leads to the following Hamilton-JacobiBellman (HJB) equation for the indirect utility function $J$, 


$$
\begin{aligned}
& \max _{\phi}\left(\frac{\phi^{2} W^{2} V}{2} J_{W W}+\phi \rho \sigma W V J_{W V}+\frac{\sigma^{2} V}{2} J_{V V}\right. \\
& \quad+(r+\phi(\eta-\mu \lambda) V) W J_{W}+(\alpha-\beta V-\kappa \lambda V) J_{V} \\
& \left.\quad+\lambda V(E[J(W(1+\phi X), V+Y, t)]-J)+J_{t}\right)=0
\end{aligned}
$$

where $J_{W}, J_{V}$, and $J_{t}$ denote the derivatives of $J(W, V, t)$ with respect to $W, V$, and $t$, and similarly for the higher derivatives, and the expectation is taken with respect to the joint distribution of $X$ and $Y$.

We solve for the optimal portfolio strategy $\phi^{*}$ by first conjecturing (which we later verify) that the indirect utility function is of the form

$$
J(W, V, t)=\frac{1}{1-\gamma} W^{1-\gamma} \exp (A(t)+B(t) V),
$$

where $A(t)$ and $B(t)$ are functions of time but not of the state variables $W$ and $V$. Given this functional form, we take derivatives of $J(W, V, t)$ with respect to its arguments, substitute into the HJB equation in equation (10), and differentiate with respect to the portfolio weight $\phi$ to obtain the following first-order condition,

$$
(\eta-\mu \lambda) V+\rho \sigma B V-\gamma \phi^{*} V+\lambda V E\left[\left(1+\phi^{*} X\right)^{-\gamma} X e^{B Y}\right]=0
$$

Before solving this first-order condition for $\phi^{*}$, it is useful to first make several observations about its structure. In particular, note that if $\lambda$ is set equal to zero, the risky asset follows a pure diffusion process. In this case, the investor faces a standard dynamic portfolio choice problem in which the first-order condition for $\phi^{*}$ becomes

$$
\eta V+\rho \sigma B V-\gamma \phi^{*} V=0
$$

Alternatively, consider the case where the investor faces a static single-period portfolio problem where the return on his portfolio during this period equals $(1+\phi X)$. In this 
case, the investor maximizes his expected utility over terminal wealth by selecting a portfolio to satisfy the first-order condition,

$$
E\left[\left(1+\phi^{*} X\right)^{-\gamma} X\right]=0
$$

Now compare the first-order conditions for the standard dynamic problem and the static buy-and-hold problem to the first-order condition for the event-risk portfolio problem given in equation (12). It is easily seen that the left-hand-side of equation (12) essentially incorporates the first-order conditions in equations (13) and (14). In the special case where $\mu$ and $Y$ equal zero, the left-hand-side of equation (12) is actually a simple linear combination of the first-order conditions in equations (13) and (14) in which the coefficients for the dynamic and static first-order conditions are one and $\lambda V$, respectively. This provides some economic intuition for how the investor views his portfolio choice problem in the event-risk model. At each instant, the investor faces a small continuous return, and with probability $\lambda V$, may also face a large return similar to that earned on a buy-and-hold portfolio over some discrete period. Thus, the first-order condition for the event-risk problem can be viewed as a blend of the first-order conditions for a standard dynamic portfolio problem and a static buy-and-hold portfolio problem.

So far, we have placed little structure of the joint distribution of the jump sizes $X$ and $Y$. To guarantee the existence of an optimal policy, however, we require that the following mild regularity conditions hold for all $\phi$ that satisfy the conditions of Proposition 1,

$$
\begin{aligned}
& M_{1} \equiv E\left[\left(1+\phi^{*} X\right)^{-\gamma} X e^{B Y}\right]<\infty, \\
& M_{2} \equiv E\left[\left(1+\phi^{*} X\right)^{1-\gamma} e^{B Y}\right]<\infty .
\end{aligned}
$$

The following proposition provides an analytical solution for the optimal portfolio strategy. 
PROPOSITION 2: Optimal Portfolio Weights. Assume that the regularity conditions in equations (15) and (16) are satisfied. Then the asset allocation problem in equations (4) and (5) has a solution $\phi^{*}$. The optimal portfolio weight is given by solving the following nonlinear equation for $\phi^{*}$,

$$
\phi^{*}=\frac{\eta-\mu \lambda}{\gamma}+\frac{\rho \sigma B}{\gamma}+\frac{\lambda M_{1}}{\gamma},
$$

subject to the constraints in (7), and where $B$ is defined by the ordinary differential equation

$$
\begin{gathered}
B^{\prime}+\sigma^{2} B^{2} / 2+\left(\phi^{*} \rho \sigma(1-\gamma)-\beta-\kappa \lambda\right) B \\
+\left(\frac{\gamma(\gamma-1) \phi^{* 2}}{2}+(\eta-\mu \lambda)(1-\gamma) \phi^{*}+\lambda M_{2}-\lambda\right)=0 .
\end{gathered}
$$

Proof: See Appendix.

From this proposition, $\phi^{*}$ can be determined under very general assumptions about the joint distribution of the jump sizes $X$ and $Y$ by solving a simple pair of equations. Given a specification for the joint distribution of $X$ and $Y$, equation (17) is just a nonlinear expression in $\phi^{*}$ and $B$. Equation (18) is an ordinary differential equation for $B$ with coefficients that depend on $\phi^{*}$. These two equations are easily solved numerically using standard finite difference techniques. Starting with the terminal condition $B(T)=0$, the values of $\phi^{*}$ and $B$ at all earlier dates are obtained by solving pairs of nonlinear equations recursively back to time zero. Given the simple forms of equations (17) and (18), the recursive solution technique is virtually instantaneous. Observe that solving this pair of equations for $\phi^{*}$ and $B$ is far easier than solving the two-dimensional HJB equation in (10) directly. For many special cases, the optimal portfolio weight can actually be solved in closed-form, or can be obtained directly by solving a single nonlinear equation in $\phi^{*}$. Several examples are presented in the next section.

We first note that the optimal portfolio weight is independent of the state variables $W$ and $V$. In other words, there is no "market timing" in either wealth or stochastic volatility. The reason why the portfolio weight is independent of wealth stems from the homogeneity of the portfolio problem in $W$. The reason the optimal 
portfolio does not depend on $V$ is formally due to the fact that we have assumed that the risk premium is proportional to $V$. Intuitively, however, this risk premium seems sensible since both the instantaneous variance of returns and the instantaneous risk of a jump are proportional to $V$; by requiring the risk premium to be proportional to $V$, we guarantee that all of the key instantaneous moments of the investment opportunity set are of the same order of magnitude.

Recall from the earlier discussion that the event-risk portfolio problem blends a standard dynamic problem with a static buy-and-hold problem. Intuitively, this can be seen from the expression for the optimal portfolio weight given in equation (17). As shown, the right-hand-side of this expression has three components. The first consists of the instantaneous risk premium $\eta-\mu \lambda$ divided by the risk aversion parameter $\gamma$. It is easily shown that when $\lambda=0$ and $V$ is not stochastic, the instantaneous risk premium become $\eta$ and the optimal portfolio policy is $\eta / \gamma$. Thus, the first term in (17) is just the generalization of the usual myopic component of the portfolio demand. The second component is directly related to the correlation coefficient $\rho$ between instantaneous returns on the risky asset and changes in the volatility $V$. When this correlation is nonzero, the investor can hedge his expected utility against shifts in $V$ by taking a position in the risky asset. Thus, this second term can be interpreted as the volatility hedging demand for the risky asset. A similar volatility hedging demand for the risky asset also appears in stochastic-volatility models such as Liu (1999). Note that in this model, the hedging demand arises not only because the state variable $V$ impacts the volatility of returns, but also because it drives the variation in the probability of an event occurring. Thus, investors have a double incentive to hedge against variation in $V$ through their portfolio holdings of the risky asset. Finally, the third term in equation (17) is directly related to the first-order condition for the static buy-and-hold problem from equation (14). Thus, this term can be interpreted as the event-risk or "illiquidity" hedging term; this term does not appear in portfolio problems where prices follow continuous sample paths.

\section{Examples and Numerical Results}

In this section, we illustrate the implications of event-related jumps for portfolio 
choice through several simple examples.

\section{A. Constant Volatility and Deterministic Jump Size}

In the first example, $V$ is assumed to be constant over time. This implies that $\alpha=\beta=\kappa=\sigma=Y=0$. In addition, we assume that price jumps are deterministic in size, implying $X=\mu$. In this case, the risky asset follows a simple jump-diffusion process. This complements Merton (1971) who studies asset allocation when the riskless asset follows a jump-to-ruin process.

In this example, the model dynamics reduce to

$$
\begin{gathered}
d S_{t}=\left(r+\eta V_{0}-\mu \lambda V_{0}\right) S_{t} d t+\sqrt{V_{0}} S_{t} d Z_{1 t}+\mu S_{t-} d N_{t} \\
d V_{t}=0 .
\end{gathered}
$$

Substituting in the parameter restrictions and solving gives the following simple expression for the optimal portfolio weight,

$$
\phi^{*}=\frac{\eta-\mu \lambda}{\gamma}+\frac{\mu \lambda}{\gamma}\left(1+\mu \phi^{*}\right)^{-\gamma}
$$

which is easily solved for $\phi^{*}$. Assuming that $\eta>0$, it is readily shown that $\phi^{*}>0$. Note that the optimal portfolio strategy does not depend on time or the investor's horizon. This occurs since the instantaneous distribution of returns does not vary over time; the instantaneous expected return, return variance, and probability of a jump are constant through time.

There are several interesting subcases for this example which are worth examining. For example, consider the subcase where $\lambda=0$, implying that the price follows a pure diffusion. In this situation, the optimal portfolio weight is simply

$$
\phi^{*}=\frac{\eta}{\gamma}
$$

Alternatively, consider the related (but non-nested) case where the price of the risky asset follows a pure jump process; where the diffusion component of the price dynamics is set equal to zero. In this situation, the optimal portfolio weight becomes 


$$
\phi^{*}=\frac{1}{\mu}\left[\left(1-\frac{\eta}{\mu \lambda}\right)^{-\frac{1}{\gamma}}-1\right]
$$

These cases make clear that the portfolio that is optimal when the price process follows a pure diffusion is very different from the optimal portfolio when the price process follows a pure jump process. When the price process follows a jump diffusion, the investor has to choose a portfolio that captures aspects of both of these special cases. Because of the nonlinearity inherent in the expression for the portfolio weight in equation (21), however, the optimal portfolio cannot be expressed as a simple linear combination or portfolio of the optimal portfolios for the two special cases given in equations (22) and (23).

Differentiating $\phi^{*}$ with respect to the parameters implies the following comparative static results,

$$
\frac{\partial \phi^{*}}{\partial \eta}>0, \quad \frac{\partial \phi^{*}}{\partial \lambda}<0, \quad \frac{\partial \phi^{*}}{\partial \gamma}<0
$$

provided $\eta>0$. Interestingly,

$$
\frac{\partial \phi^{*}}{\partial \mu}>0, \quad \text { if } \mu<0, \quad \frac{\partial \phi^{*}}{\partial \mu} \leq 0, \quad \text { if } \mu \geq 0 .
$$

To illustrate this result, the top graph in Figure 1 plots the optimal portfolio weight as a function of the value of the jump size $\mu$. As shown, the optimal portfolio weight is highly sensitive to the size of the jump $\mu$. When the jump is in the downward direction, the investor takes a smaller position in the risky asset than he would if jumps did not occur. Surprisingly, however, the investor also takes a smaller position when the jump is in the upward direction. The rationale for this is related to the effects of jumps on the variance and skewness of the distribution of terminal wealth. Holding fixed the risk premium, jumps in either direction increase the variance of the distribution. On the other hand, jumps also affect the skewness (and other higher moments) of the return distribution and the investor benefits from positive skewness. Despite this, the variance effect dominates and the investor takes a smaller position 
in the risky asset for nonzero values of $\mu$. The skewness effect, however, explains why the graph of $\phi^{*}$ against $\mu$ is asymmetric.

To illustrate just how different portfolio choice can be in the presence of event risk, the second graph in Figure 1 plots the optimal portfolio as a function of the risk aversion parameter $\gamma$ for various jump sizes $\mu$. When $\mu=0$ and no jumps occur, the investor takes an unboundedly large position in the risky asset as $\gamma \rightarrow 0$. In contrast, when there is any risk of a downward jump, the optimal portfolio weight is bounded above as $\gamma \rightarrow 0$. This feature is a simple implication of Proposition 1, but serves to illustrate that the optimal portfolio in the presence of event risk is qualitatively different from the optimal portfolio when event risk is not present.

This also makes clear that the optimal strategy is not driven purely by the effects of jumps on return skewness and kurtosis. For example, skewness and kurtosis effects are also present in models where volatility is stochastic and correlated with risky asset returns, but jumps do not occur. In these models, however, investors do not place bounds on their portfolio weights of the type described in Proposition 1. Furthermore, the optimal portfolio in these models does not involve any static buy-and-hold component. This underscores the point that many of the features of the optimal portfolio strategy in our framework are uniquely related to the event risk faced by the investor.

To provide some specific numerical examples, Table I reports the value of $\phi^{*}$ for different values of the parameters. In this table, the risk premium for the risky asset is held fixed at seven percent and the standard deviation of the diffusive portion of risky asset returns is held fixed at 15 percent. As shown, relative to the benchmark where $\mu=0$, the optimal portfolio weight can be significantly less even when the probability of an event occurring is extremely low. For example, even when a negative 90 percent jump occurs at a 100-year frequency, the portfolio weight is typically much less than 50 percent of what it would be without jumps. Note that this effect is not symmetric; a positive 90 percent jump at a 100-year frequency has a much smaller effect on the portfolio weight. Also observe that the effects of jumps on portfolio weights are much more pronounced for investors with lower levels of risk aversion. This counterintuitive effect occurs because less-risk-averse investors would prefer to hold more leveraged 
positions, but cannot because they do not have full control over their portfolio. Thus, the effects of event risk fall much more heavily on investors with low levels of risk aversion who would otherwise be more aggressive.

\section{B. Stochastic Volatility and Deterministic Jump Sizes}

In the second example, $V$ is also allowed to follow a jump-diffusion process. The two jump sizes $X$ and $Y$, however, are assumed to be constants with values $\mu$ and $\kappa$, respectively. The jump size $\mu$ can be positive or negative. The jump size $\kappa$ can only be positive.

In this example, the model dynamics become

$$
\begin{gathered}
d S_{t}=\left(r+\eta V_{t}-\mu \lambda V_{t}\right) S_{t} d t+\sqrt{V_{t}} S_{t} d Z_{1 t}+\mu S_{t-} d N_{t} \\
d V_{t}=\left(\alpha-\beta V_{t}-\kappa \lambda V_{t}\right) d t+\sigma \sqrt{V_{t}} d Z_{2 t}+\kappa d N_{t} .
\end{gathered}
$$

Applying the results in Proposition 2 to this model gives the following expression for the optimal portfolio weight

$$
\phi^{*}=\frac{\eta-\mu \lambda}{\gamma}+\frac{\rho \sigma B}{\gamma}+\frac{\lambda \mu}{\gamma}\left(1+\mu \phi^{*}\right)^{-\gamma} e^{\kappa B}
$$

which can be solved for $\phi^{*}$ jointly with the equation for $B$ given in equation (18).

Because of the dependence on $B$, the optimal portfolio weight is now explicitly a function of the investor's investment horizon. Examining equation (28) indicates that there are several ways in which the investment horizon affects the optimal portfolio weight. Specifically, $B$ appears in conjunction with the correlation coefficient $\rho$ reflecting that there is a dynamic hedging component to the investor's demand for the risky asset. Since $V$ is mean reverting, the horizon over which investment decisions are made is important. However, dynamically hedging shifts in $V$ is not the only reason why there is time dependence in the optimal portfolio weight. For example, when $\rho=0$, the risky asset cannot be used to hedge against shifts in the investment opportunity set arising from variation in $V$. Despite this, the optimal portfolio weight still depends on the investor's horizon through the $e^{\kappa B}$ term in equation (28). Thus, 
time dependence enters the problem both through the dynamic hedging component as well as through the static hedging component.

The top graph in Figure 2 plots the optimal portfolio weight as a function of the investor's horizon for various values of the dynamic hedging parameter $\rho$. In this case, $\phi^{*}$ is an increasing function of the horizon for each of the values of $\rho$ plotted. We note, however, that $\phi^{*}$ can be a decreasing function of the investor's horizon when $\gamma<1$. This graph also illustrates that the optimal portfolio weight converges to a constant as $T \rightarrow \infty$. Furthermore, the dependence of the optimal portfolio weight on $\rho$ indicates that an important part of the demand for the risky asset comes from its ability to dynamically hedge the continuous portion of changes in $V$.

An important feature of this event-risk model is that both prices and volatility are allowed to jump. The previous section illustrated that the presence of price jumps in either direction induces investors to take smaller positions in the risky asset. Intuitively, one might suspect that introducing jumps in volatility would have a similar effect on the optimal portfolio weight. Surprisingly, this is not true in general. This can be seen from the second graph in Figure 2 which plots the optimal portfolio weight as a function of the size of the volatility jump $\kappa$ for different values of $\mu$. As shown, the optimal portfolio weight can be an increasing function of $\kappa$ for some values of $\mu$.

This result illustrates the important point that in addition to its ability to dynamically hedge against continuous changes in $V$, the risky asset can also be used as a static hedge against the effects of jumps in $V$. This second hedging role is one that does not occur in traditional portfolio choice models where state variables have continuous sample paths. The fact that the risky asset can be used to hedge in two different ways, however, makes it evident that the investor faces a dilemma in choosing a portfolio strategy. In particular, the portfolio that hedges against small local diffusion-induced changes in the state variables is not the same as the portfolio that hedges against large jumps in the state variables. This problem is inherent in the fact that when there is event risk, the portfolio problem has features of both a dynamic portfolio problem and an illiquid buy-and-hold problem.

Finally, if we impose the parameter restrictions $\rho=0$ and $\kappa=0$, volatility is 
still stochastic but the optimal portfolio weight becomes the same as in Section III.A. where volatility is not stochastic. Thus, continuous stochastic variation in $V$ only affects the optimal portfolio weight if it is hedgable through a nonzero value of $\rho$.

\section{Implications For Optimal Portfolio Choice}

Moving beyond the numerical examples presented in Section III, it is useful to explore how event risk might affect the optimal portfolio of an investor in a specific market. To this end, we calibrate the model to be roughly consistent with historical stock index returns and stock index return volatility in the U.S. To make this process as straightforward as possible, we focus on the simple stochastic volatility model with deterministic jump sizes described in section III. Once calibrated to U.S. data, we explore the key implications of the model for investors.

In parameterizing this model of event risk, it is important to recognize that the major financial events addressed by our model are infrequent by their nature. Ideally, we would like to use a calibration approach that minimizes the effects of the inherent "Peso problem" on the results. Although there are many ways to do this, we use the following informal (but hopefully intuitive) approach to allow us to estimate the size and frequency of events from the longest time series available. ${ }^{10}$

We first obtain the monthly return series for U.S. stocks during the 1802 to 1925 period created and described in Schwert (1990). We then append the CRSP monthly value-weighted index returns for the 1926 to 2000 period to give a time series of returns spanning nearly 200 years. A review of the data shows that there are eight observations where the stock index dropped by 20 or more percent. These observations include the beginning of the Civil War in May 1861, the black Friday crash of October 1929, and the October 1987 stock market crash. Interestingly, four of the eight observations are clustered in the high-volatility decade of the 1930s, consistent with the double-jump model. Since these observations are roughly five standard deviations below the mean, it is not unreasonable to view these negative returns as being due to a jump event. A back-of-the-envelope calculation suggests calibrating the model to allow a -25 percent jump (the average of the eight observations) at 
an average frequency of about 25 years. To provide a rough estimate of the size of the volatility jump, we compute the standard deviation of returns for the five-month window centered at the event month. The average of these standard deviation estimates is just under 50 percent. Given this, we make the simplifying assumption that when a jump occurs, the volatility of the stock return jumps by an amount equal to the difference between 50 percent and its mean value.

The remaining parameter estimates are obtained from Table 1 of Pan (2002). Using S\&P 500 stock index returns and stock index option prices, Pan estimates the parameters of several versions of a jump-diffusion model. For simplicity, we use the parameter values Pan estimates for her SV0 model, and adjust them slightly to be consistent with our estimates of jump sizes and frequencies. ${ }^{11}$ Specifically, we use Pan's estimates of $\beta=5.3$ and $\rho=-.57$. To obtain estimates of $\alpha, \eta$, and $\sigma$, we note that in our model, the expected instantaneous equity premium is $\alpha \eta / \beta$, the expected instantaneous variance of returns is $\alpha\left(1+\mu^{2} \lambda\right) / \beta$, and the the expected instantaneous variance of changes in $V$ is $\alpha\left(\sigma^{2}+\kappa^{2} \lambda\right) / \beta$. Setting these three moments equal to the corresponding estimates of .1065, .0242, and .3800 from Table 1 of Pan provides us with three equations which can be solved for the values of $\alpha, \eta$, and $\sigma$. By doing this, we guarantee that the calibrated model matches the moments of returns and volatility estimated by Pan. This approach leads to the following parameter values for the baseline case where jumps occur with an average frequency of 25 years: $\alpha=.11512, \beta=5.3000, \sigma=.22478, \eta=4.90224, \rho=-.57000, \mu=-.25000$, $\kappa=.22578$, and $\lambda=1.84156$.

To illustrate the effects that event risk has on the optimal portfolio choice for an investor where the model is calibrated to historical U.S. returns in this manner, Table II reports the portfolio weights for various levels of investor risk aversion. To facilitate comparison, we report the portfolio weights for the case where there are no jumps, where there are only jumps in the stock index, and the baseline case where there are jumps in both the stock index and volatility. Note that for the nonbenchmark cases, we recalibrate the model so that we match the expected instantaneous moments estimated by Pan (2002) using the procedure described in the previous paragraph. In each case, the investor has a five-year investment horizon. 
Table II shows that the possibility of a 25 percent downward jump in stock prices has an important effect on the investor's optimal portfolio, even though this type of event happens only every 25 years on average. For example, the optimal portfolio weight for an investor with a risk aversion parameter of two is 2.305 if no jumps can occur, is 1.929 if only jumps in the stock price can occur, and is 2.010 if both jumps in stock prices and volatility can occur. Observe that from Proposition 1, the investor never takes a position in the risky asset greater than four since jumps of -25 percent can occur. Table II shows that the risk of a downward jump always induces the investor to take a smaller position in the stock market than he would otherwise.

Table II also makes clear that while jumps in volatility do not have as much of an effect as jumps in the stock price, they do have an important influence on the optimal portfolio. Interestingly, jumps in volatility decrease the optimal portfolio weight when $\gamma<1$, and increase the optimal portfolio weight when $\gamma>1$. Intuitively, the reason for this is related to the effect of a volatility jump on the distribution of the investor's returns. Recall that in this model, the instantaneous Sharpe ratio of returns is increasing in the volatility $V$ because of the form of the risk premium. Thus, when an event occurs, the investor suffers an immediate loss because of the downward jump in the stock price, but then faces an improved risk-return tradeoff because the jump in volatility increases the Sharpe ratio. This pattern induces a type of negative serial correlation or smoothness into the time series of the investor's returns which can be shown to reduce both the first and second moments of the distribution of the investor's terminal wealth. As shown by Samuelson (1991), however, investors who are less risk averse than logarithmic $(\gamma<1)$, will reduce their portfolio weight as this smoothing increases while the opposite is true for investors who are more risk averse than logarithmic. Thus, an increase in the volatility jump size parameter $\kappa$ leads to a decrease in the portfolio weight for $\gamma<1$, and to an increase in the portfolio weight for $\gamma>1$. Another way of seeing this is to note that for $\gamma>1$, the investor's utility is unbounded from below as his wealth approaches zero. Thus, the investor is particularly averse to a run of successive negative returns. Since a jump in $V$ reduces the likelihood of a run of negative returns, the investor with $\gamma>1$ is more confident and takes a larger stock position. In contrast, for $\gamma<1$, the investor's utility is bounded from below but unbounded from above. Thus, the investor benefits less 
from the reduction in variance of the distribution of terminal wealth and reduces his portfolio weight because of the reduction in the first moment. ${ }^{12}$

Another interesting issue to consider is the loss suffered by an investor who does not consider the effects of price and volatility jumps in making portfolio decisions. ${ }^{13}$ To examine this, we do the following. Assume that there is an investor who believes that there are no jumps, implying that $\lambda=\mu=\kappa=0$. This investor calibrates his model to match the moments using the procedure described earlier. Given this calibration, the investor then follows the portfolio strategy that would be optimal if $\lambda=\mu=\kappa=0$. Let us denote this strategy $\hat{\phi}$. Now assume that there are actually jumps in prices and volatility. In this situation, the optimal portfolio weight $\phi^{*}$ differs from $\hat{\phi}$, and the investor suffers a loss by following this strategy. Following a procedure similar to that used to solve for $J(W, V, t)$, we can solve for the investor's utility of wealth function when he follows strategy $\hat{\phi}$. Denote this utility of wealth function $K(W, V, t)$. Because $\hat{\phi}$ is suboptimal, it is clear that $K(W, V, t)<J(W, V, t)$. To quantify the loss, we assume that this investor following the suboptimal strategy starts with $W=1$, and solve for the $\hat{W}$ such that an investor with $W=\hat{W}$ who followed the optimal strategy would attain the same level of utility. Specifically, this utility equivalent wealth $\hat{W}$ is obtained by solving numerically the equation $J(\hat{W}, V, t)=K(1, V, t)$. Note that the utility equivalent wealth $\hat{W}$ is less than or equal to one since following the suboptimal strategy $\hat{\phi}$ reduces the utility of the investor's wealth. Finally, we calculate the loss using this wealth-based metric by taking the difference $1-\hat{W}$ and converting it into percentage terms by multiplying by 100 . We designate this metric the wealth equivalent loss.

Table II reports the wealth equivalent losses for an investor who does not consider the effects of jumps. There are several key features shown in Table II. First, when the suboptimal strategy $\hat{\phi}$ exceeds the bound in Proposition 1, then a jump to ruin is possible and clearly, $K(W, V, t)=-\infty$. In these cases, it is clear that the wealth equivalent loss of following the suboptimal strategy is 100 percent; the investor obtains the same expected utility that he would if he had no wealth at all. Second, Table II shows that the wealth equivalent loss can be nontrivial for other ranges of the risk aversion parameter. Specifically, when $\gamma=2.00$, the wealth equivalent loss is 3.2 
percent when only price jumps can occur, and is 2.0 percent when both price and volatility jumps can occur. Table II also shows that the wealth equivalent loss is a decreasing function of $\gamma$.

Although we have calibrated the model to historical U.S. returns, it is important to recognize that U.S. returns may not fully reflect the size of potential jump events. The reason for this is the possibility of a survivorship bias since the U.S. has experienced historically high returns. This point is also consistent with Jorion and Goetzmann (1999) who show that many countries have experienced huge market declines during relatively short periods of time during the past century. In many cases, major events such as wars or political crises have actually led to stock markets being closed for years (or even decades). These closures have often resulted in catastrophic losses for investors. To reflect this downside risk to financial markets, we also consider a scenario where stock market jumps of -50 percent and volatility jumps to 70 percent occur at an average frequency of 100 years. Following the same calibration approach as before implies parameter values for this scenario of $\alpha=.11512$, $\beta=5.3000, \sigma=.21099, \eta=4.90224, \rho=-.57000, \mu=-.50000, \kappa=.46578$, and $\lambda=.46039$.

Table III reports the optimal portfolio weights for this alternative scenario. Even though the frequency of an event is much less, it has an even larger effect on the optimal portfolio weight than in Table II. For example, the optimal portfolio weight for an investor with a risk aversion parameter of two is still 2.305 if no jumps can occur. If only jumps in the stock price can occur, then the portfolio weight is now 1.395 rather than 1.929. If both jumps in the stock price and volatility can occur, the optimal portfolio weight is now 1.481 rather than the value of 2.010 given in Table II. As before, jumps in volatility decrease the optimal portfolio weight for $\gamma<1$, and vice versa.

Table III also reports the corresponding wealth equivalent losses. As in Table II, the wealth equivalent loss can be 100.0 percent when the bound given in Proposition 1 is violated. It is interesting to note, however, that there is a case shown in Table III where an investor following the suboptimal strategy attains $K(W, V, t)=-\infty$ even when $\hat{\phi}$ does not violate the bound given Proposition 1. Specifically, when $\gamma=3.00$, 
an investor who does not consider the effects of volatility jumps has a portfolio weight of 1.564 (which does not violate the bound) but still has a wealth equivalent loss of 100.0 percent when both price and volatility jumps can occur. Intuitively, this occurs because the finiteness of the expected utility function can only be guaranteed when the optimal strategy $\phi^{*}$ is followed. ${ }^{14}$ Specifically, when the suboptimal portfolio weight $\hat{\phi}$ is sufficiently high (but still less than the bound given in Proposition 1), the return distribution for the investor's wealth may be such that the expectation of his terminal utility equals $-\infty$. For example, consider the case where $\gamma=2$, and expected utility equals $-E\left[\frac{1}{W_{T}}\right]$. Even though all positive moments of the distribution of $W_{T}$ are finite when $\hat{\phi}$ is followed, the expectation $E\left[\frac{1}{W_{T}}\right]$ may fail to exist, implying $K(W, V, t)=-\infty$. Thus, even when a jump to complete ruin cannot occur, a strategy may be so suboptimal that the investor has a wealth equivalent loss of 100 percent. ${ }^{15}$ Another way of seeing this is by considering the case where the stock price jump is -50 percent. By following a strategy where $\phi$ is less than two, ruin can be avoided. However, imagine that $\hat{\phi}$ is close to two, say 1.99. If a jump occurs, the investor will clearly lose virtually all of his wealth. After the jump, however, the investor would rebalance his portfolio to attain $\hat{\phi}=1.99$ again. Thus, if another jump occurs, the investor's remaining wealth will again be virtually eliminated. The key point is that even though total ruin does not occur, the resulting distribution of $W_{T}$ has enough mass in the neighborhood of zero that the expected utility function need not be finite. When $\phi$ is more distant from the bound in Proposition 1, as is the case for $\phi^{*}$, this situation does not arise and expected utility is finite.

Finally, Table III shows the wealth equivalent losses can be significant for other parameter values. For example, when only price jumps can occur, an investor with $\gamma=3.00$ who ignores the effects of jumps has a wealth equivalent loss of 30.5 percent. For larger values of $\gamma$, the wealth equivalent losses are smaller, but are still economically significant.

The results in Tables II and III are based on two simple calibrations of the model. Given than there is always uncertainty about the precise values of estimated parameters, however, it is useful to provide some additional information about the sensitivity of the optimal portfolio weights to the key jump size and frequency parameters. To 
this end, Table IV reports the optimal portfolio weights for various combinations of jump frequencies and price jump sizes, while Table $\mathrm{V}$ reports the optimal portfolio weights for various combinations of jump frequencies and volatility jump sizes. For each set of jump size and frequency parameters in these two tables, the $\alpha, \eta$, and $\sigma$ parameters are chosen to match the three moments from Table 1 of Pan (2002) using the same procedure as before. We note that in a few cases involving large but infrequent jumps, these moments cannot be matched since they imply negative values for $\sigma$; these cases are designated by a dash in Tables IV and V.

Tables IV and V indicate that the optimal portfolio weight is clearly affected by both the jump size and frequency parameters. The size of the price jump appears to have the largest effect on the optimal portfolio weight. The size of the volatility jump as well as the level of the frequency parameter can also have important effects. Despite this dependence on the parameter values, however, Table IV and V indicate that the optimal portfolio weight is generally fairly robust to small perturbations in the parameter values. This is important since it implies that even if the jump size and frequency parameters are estimated with some error (provided it is not overly large) from historical data, the general implications for optimal portfolio choice may still be qualitatively valid.

Admittedly, we have focused only on simple calibrations of one of the simplest versions of the model. Despite this, however, we believe that several important general insights about the role that event risk could play in real-world portfolio decisions emerge from this analysis. Foremost among these is that investors have strong incentives to significantly reduce their exposure to the stock market when they believe that there is event risk. This is true even when the probability of a major downward jump in stock prices is very small, as in the scenario of a -50 percent jump occurring every 100 years on average. Certainly, jumps of this magnitude and frequency cannot be ruled out; it is all too easy to think of extreme situations where a downward jump of this magnitude could occur during the next century even in the U.S., particularly in the wake of September 11th, 2001. Our analysis suggests a possible reason why historical levels of participation in the stock market have been much lower than standard portfolio choice models would view as optimal. ${ }^{16}$ 


\section{Conclusion}

In this paper, we study the effects of event-related jumps in prices and volatility on investment strategies. Using the double-jump framework of Duffie, Pan, and Singleton (2000), we take advantage of the affine structure of the model to provide analytical solutions to the optimal portfolio problem.

The presence of event risk changes the standard portfolio problem in several important ways. First, since the investor no longer has complete control over his wealth, the investor acts as if some part of his portfolio consists of illiquid assets and he is much less willing to take leveraged or short positions. The optimal portfolio strategy blends elements of both a standard dynamic hedging strategy and a buy-and-hold or "illiquidity" hedging strategy. Furthermore, event risk affects investors with low levels of risk aversion more than it does highly risk-averse investors. These results illustrate that the implications of event risk for the optimal portfolio strategy are both subtle and complex. Our analysis suggests that jumps in both prices and volatility have important effects on optimal portfolios, although our calibrated exercise indicates that price jumps tend to have a larger effect than do volatility jumps. Finally, our results suggest that if market participants believe that there is even a remote chance of a sudden market collapse, their portfolio behavior could be very different from that implied by classical portfolio choice models which abstract from event risk.

This paper is only a first attempt to systematically study the effect of event risk on optimal portfolio choice. Along with other studies in the field of asset allocation, we use a partial equilibrium approach by taking prices as given. Clearly, however, an equilibrium study would be necessary to provide a complete understanding of the interaction between price dynamics and investor's portfolio choices. Nevertheless, we hope that this partial equilibrium study provides some understanding of the complete picture. 


\section{Appendix: Proofs}

Proof of Proposition 1: Let $\left\{W_{t}^{*}, 0 \leq t \leq T\right\}$ be the wealth process attained by an investor who follows the optimal portfolio process $\phi^{*}$. We first remark that $W_{T}^{*}$ must be positive almost surely. Otherwise, a nonzero probability of $W_{T}^{*} \leq 0$ will result in $E\left[U\left(W_{T}^{*}\right)\right]=-\infty$, which is inferior to investing all of the positive initial wealth in the riskless asset.

We next show that for $W_{T}^{*}$ to be positive almost surely, $W_{t}^{*}$ must be positive almost surely for any $t<T$. To see this, we first condition on the event that there is no jump between $t$ and $T$. This implies

$$
W_{T}=W_{t} \exp \left(\int_{t}^{T}\left(r+\phi_{\tau}(\eta-\mu \lambda) V_{\tau}-\frac{\phi_{\tau}^{2} V_{\tau}}{2}\right) d \tau+\phi_{\tau} \sqrt{V_{\tau}} d Z_{1}\right)
$$

for any portfolio policy $\phi$. Such an event of no jump between $t$ and $T$ has a positive probability given the assumption that

$$
0<E_{t}\left[\exp \left(-\int_{t}^{T} \lambda V_{\tau} d \tau\right)\right]<1
$$

So $W_{T}^{*}>0$ almost surely implies $W_{t}^{*}>0$ almost surely for any $t$.

Finally, we show that for $W_{t}^{*}>0$ almost surely, the optimal portfolio weight $\phi^{*}$ must satisfy equation (7). Suppose equation (7) is not satisfied for some $t$. Then there is a positive probability of a jump event between $t$ and $t+\Delta t$ for some $\Delta t>0$. Conditioning on such a jump event, the time- $t$ wealth is $W_{t}=W_{t-}(1+\phi X)$, where $W_{t-}$ is the wealth before the jump event, and where $X$ is the jump size. By the definition of $X_{\text {Inf }}$ and $X_{\text {Sup }}$, we have for an arbitrary $\epsilon>0$, a positive probability of $X \in\left(X_{\mathrm{Inf}}, X_{\mathrm{Inf}}+\epsilon\right)$ and a positive probability of $X \in\left(X_{\mathrm{Sup}}-\epsilon, X_{\mathrm{Sup}}\right)$. Thus, if (7) is not satisfied, there is a positive probability of $W_{t}^{*} \leq 0$, which contradicts the assumption that $W_{t}^{*}$ is the wealth process generated by the optimal portfolio weight $\phi^{*}$. 
Proof of Proposition 2: Suppose that the indirect utility function $J$ is of the conjectured form in equation (11) with state-independent time-varying coefficients $A(t)$ and $B(t)$ to be determined shortly. Then the first-order condition of the HJB equation (10) implies

$$
\begin{aligned}
\phi_{t}^{*} & =-\frac{J_{W}}{W J_{W W}}\left((\eta-\mu \lambda)+\rho \sigma \frac{J_{W V}}{J W}+\lambda M_{1} \frac{J}{W J_{W}}\right) \\
& =\frac{\eta-\mu \lambda}{\gamma}+\frac{\rho \sigma B}{\gamma}+\frac{\lambda M_{1}}{\gamma}
\end{aligned}
$$

which is the optimal portfolio weight given in (17). It should be noted that $\phi^{*}$ is state independent and a nonlinear function of $B$.

We now proceed to derive the ordinary differential equations for the time-varying coefficients $A(t)$ and $B(t)$, under which the conjectured form (11) for the indirect utility function $J$ indeed satisfies the HJB equation (10). For this, we substitute (11) and (12) into the HJB equation and obtain,

$$
\begin{gathered}
-\frac{\gamma \phi^{* 2} V}{2}+\phi^{*} \rho \sigma B V+\frac{\sigma^{2} B^{2} V}{2(1-\gamma)}+\left(r+\phi^{*}(\eta-\mu \lambda) V\right) \\
+(\alpha-\beta V-\kappa V) \frac{B}{1-\gamma}+\frac{\lambda V}{1-\gamma} M_{2}-\frac{\lambda V}{1-\gamma}+\frac{1}{1-\gamma}\left(A^{\prime}+B^{\prime} V\right)=0 .
\end{gathered}
$$

The left-hand-side of this expression is an affine function in $V$. For this expression to hold for all $V$, the constant term and the linear coefficient of $V$ on the left-hand-side must be set equal to zero separately, which leads to the ordinary differential equation for $B(t)$ given in (18) and the following ordinary differential equation for $A(t)$

$$
A^{\prime}+\alpha B+(1-\gamma) r=0
$$




\section{FOOTNOTES}

${ }^{1}$ For example, the VIX index of S\&P 500 stock index option implied volatilities increased 313 percent on October 19, 1987, 53 percent on October 27, 1997, and 28 percent on August 27, 1998.

${ }^{2}$ For example, see the extensive recent study by Eraker, Johannes, and Polson (2000) of the double-jump model.

${ }^{3} \mathrm{Wu}(2000)$ studies the portfolio choice problem in a model where there are jumps in stock prices but not volatility, but does not provide a verifiable analytical solution for the optimal portfolio strategy.

${ }^{4}$ Although the risk premium could be separated into the two types of risk premia, the portfolio allocation between the riskless asset and the risky asset in our model is independent of this breakdown. If options were introduced into the market as a second risky asset, however, this would no longer be true (see Pan (2002)).

${ }^{5}$ See Chacko and Viceira (2000) and Longstaff (2001) for solutions to the dynamic portfolio problem for alternative stochastic volatility models.

${ }^{6}$ Similar evidence is also presented in Bates (2000), Pan (2002), and others.

${ }^{7}$ See also Bakshi, Cao, and Chen (1997) and Bates (2000) for empirical evidence about the importance of jumps in option pricing.

${ }^{8}$ Imposing buy-and-hold constraints on an otherwise dynamic trading strategy parallels our jump-induced constraint. Haugh and Lo (2001) show that options can alleviate some of the cost associated with the buy-and-hold constraint.

${ }^{9}$ We thank the referee for pointing out the role that options might play in mitigating the effects of event risk.

${ }^{10}$ We note that although it is beyond the scope of this paper, the general doublejump model could be formally estimated using either the efficient method of moments (EMM) approach applied by Andersen, Benzoni, and Lund (2001) or the Monte Carlo Markov chain (MCMC) technique used by Eraker, Johannes, and Polson (2000).

${ }^{11}$ The advantage of using the parameter estimates for Pan's SV0 model is that 
they represent parameter estimates for the stochastic volatility model in the absence of jumps. This then allows us to calibrate the model for different jump sizes using a particularly simple algorithm. As pointed out by Pan, allowing for jumps significantly enhances the ability of the stochastic volatility model to capture the properties of the data.

${ }^{12}$ Consistent with this intuition, when both stock price and volatility jumps are positive, the effect of an increase in the volatility jump size parameter $\kappa$ is reversed. In particular, the portfolio weight is then an increasing function of $\kappa$ for $\gamma<1$, and vice versa.

${ }^{13} \mathrm{We}$ are grateful to the referee for raising this issue.

${ }^{14}$ Note that in this case, $\gamma=3$, which means that utility is unbounded from below.

${ }^{15}$ This feature appears in many other continuous time portfolio choice models and is not unique to jump diffusion models. For other examples, see Liu (1999).

${ }^{16}$ For example, see Mankiw and Zeldes (1991), Heaton and Lucas (1997), and Basak and Cuoco (1998). 


\section{REFERENCES}

Aase, Knut K., 1984, Optimum portfolio diversification in a general continuous-time model, Stochastic Processes and Their Applications 18, 81-98.

Aase, Knut K., 1986, Ruin problems and myopic portfolio optimization in continuous trading, Stochastic Processes and Their Applications 21, 213-227.

Aase, Knut K., and Bernt Øksendal, 1988, Admissible investment strategies in continuous trading, Stochastic Processes and Their Applications 30, 291-301.

Andersen, Tobin G., Luca Benzoni, and Jesper Lund, 2001, An empirical investigation of continuous-time equity return models, Journal of Finance, forthcoming.

Ang, Andrew, and Geert Bekaert, 2000, International asset allocation with time-varying correlations, Review of Financial Studies, forthcoming.

Bakshi, Gurdip, Charles Cao, and Zhiwu Chen, 1997, Empirical performance of alernative option pricing models, Journal of Finance 52, 2003-2049.

Balduzzi, Pierluigi, and Anthony W. Lynch, 1999, Transaction costs and predictability: Some utility cost calculations, Journal of Financial Economics 52, 47-78.

Barberis, Nicholas, 2000, Investing for the long run when returns are predictable, Journal of Finance 55, 225-264.

Bardhan, Indrajit, and Xiuli Chao, 1995, Martingale analysis for assets with discontinuous returns, Mathematics of Operations Research 20, 243-256.

Basak, Suleyman, and Domenico Cuoco, 1998, An equilibrium model with restricted stock participation, Review of Financial Studies 11, 309-341.

Bates, David, 2000, Post-' 87 crash fears in S\&P 500 futures options, Journal of Econometrics 94, 181-238. 
Brennan, Michael, Eduardo Schwartz, and Ronald Lagnado, 1997, Strategic asset allocation, Journal of Economic Dynamics and Control 21, 1377-1403.

Brennan, Michael, and Yihong Xia, 1998, Resolution of a financial puzzle, Working paper, UCLA.

Campbell, John, and Luis Viceira, 1999, Consumption and portfolio decisions when expected returns are time varying, The Quarterly Journal of Economics 114, 433-495.

Campbell, John, and Luis Viceira, 2001, Who should buy long-term bonds? American Economic Review 91, 99-127.

Chacko, George, and Luis Viceira, 2000, Dynamic consumption and portfolio choice with stochastic volatility in incomplete markets, Working paper, Harvard University.

Common, Etienne, 2000, Extreme events and the role of learning in financial markets, Working paper, Harvard University.

Das, Sanjiv R., and Raman Uppal, 2001, Systemic risk and international portfolio choice, Working paper, Santa Clara University.

Duffie, Darrell, Jun Pan, and Kenneth Singleton, 2000, Transform analysis and asset pricing for affine jump diffusion, Econometrica 68, 1343-1376.

Dybvig, Philip H., and Chi-Fu Huang, 1988, Nonnegative wealth, absence of arbitrage, and feasible consumption plans, Review of Financial Studies 1, 377-401.

Eraker, Bjorn, Michael S. Johannes, and Nicholas G. Polson, 2000, The impact of jumps in returns and volatility, Working paper, Columbia University.

Harrison, J. Michael, and David M. Kreps, 1979, Martingales and arbitrage in multiperiod securities markets, Journal of Economic Theory 20, 381-408.

Haugh, Martin, and Andrew Lo, 2001, Asset allocation and derivatives, Quantitative Finance 1, 45-72. 
Heaton, John, and Deborah Lucas, 1997, Market frictions, savings behavior, and portfolio choice, Macroeconomic Dynamics 1, 76-101.

Heston, Steven, 1993, A closed-form solution of options with stochastic volatility with applications to bond and currency options, Review of Financial Studies 6, 327-343.

Jeanblanc-Picqué, Monique, and Monique Pontier, 1990, Optimal portfolio for a small investor in a market model with discontinuous prices, Applied Mathematics and Optimization 22, 287-310.

Jorion, Philippe, and William N. Goetzmann, 1999, Global stock markets in the Twentieth Century, Journal of Finance 54, 953-980.

Kim, Tong S., and Edward Omberg, 1996, Dynamic nonmyopic portfolio behavior, Review of Financial Studies 9, 141-161.

Kraus, Alan, and Robert Litzenberger, 1976, Skewness preference and the valuation of risky assets, Journal of Finance 31, 1085-1100.

Liu, Jun, 1999, Portfolio selection in stochastic environments, Working paper, UCLA.

Longstaff, Francis A., 2001, Optimal portfolio choice and the valuation of illiquid assets, Review of Financial Studies 14, 407-431.

Lynch, Anthony, 2001, Portfolio choice and equity characteristics: Characterizing the hedging demand induced by return predictability, Journal of Financial Economics 62, 67-130.

Mankiw, Gregory, and Stephen Zeldes, 1991, The consumption of stockholders and nonstockholders, Journal of Financial Economics 29, 97-112.

Merton, Robert C., 1971, Optimum consumption and portfolio rules in a continuous-time model, Journal of Economic Theory 3, 373-413.

Merton, Robert C., 1980, On estimating the expected return on the market: An exploratory investigation, Journal of Financial Economics 8, 232-361. 
Pan, Jun, 2002, The jump-risk premia implicit in option prices: Evidence from an integrated time-series study, Journal of Financial Economics 63, 3-50.

Samuelson, Paul, 1991, Long-run risk tolerance when equity returns are mean regressing: Pseudoparadoxes and vindication of "businessman's risk," in William C. Brainard, William D. Nordhaus, and Harold W. Watts, eds.: Money, Macroeconomics, and Economic Policy (M.I.T. Press, Cambridge, MA).

Schroder, Mark, and Costis Skiadas, 1999, Optimal consumption and portfolio selection with stochastic differential utility, Journal of Economic Theory 89, 68-126.

Schwert, G. William, 1990, Index of United States stock prices from 1802 to 1987, Journal of Business 63, 399-426.

Wachter, Jessica, 1999, Risk aversion and allocation to long-term bonds, Working paper, New York University.

Wu, Liuren, 2000, Jumps and dynamic portfolio decisions, Working paper, Fordham University.

Xia, Yihong, 2001, Learning about predictability: The effect of parameter uncertainty on dynamic asset allocation, Journal of Finance 56, 205-246. 
Table I

\section{Portfolio Weights with Constant Volatility and Deterministic Price Jump Sizes}

This table reports the portfolio weights for the risky asset in the case where the volatility of the asset's returns is constant and the percentage size of the jump in the asset's price is also constant. The risk premium for the risky asset is held fixed at seven percent and the volatility of diffusive returns is held fixed at 15 percent throughout the table. The frequency of jumps is expressed in years and equals the reciprocal of the jump intensity.

\begin{tabular}{|c|c|c|c|c|c|c|}
\hline \multirow{2}{*}{$\begin{array}{r}\text { Risk } \\
\text { Aversion } \\
\text { Parameter }\end{array}$} & \multirow{2}{*}{$\begin{array}{l}\text { Frequency } \\
\text { of Jumps }\end{array}$} & \multicolumn{5}{|c|}{ Percentage Jump Size } \\
\hline & & -90 & -20 & 0 & 20 & 90 \\
\hline \multirow[t]{5}{*}{.50} & 1 & .151 & 1.795 & 6.222 & 2.736 & .189 \\
\hline & 2 & .269 & 2.511 & 6.222 & 3.970 & .411 \\
\hline & 5 & .508 & 3.431 & 6.222 & 5.161 & 1.234 \\
\hline & 10 & .721 & 4.008 & 6.222 & 5.662 & 2.600 \\
\hline & 100 & 1.091 & 4.927 & 6.222 & 6.163 & 5.744 \\
\hline \multirow[t]{5}{*}{1.00} & 1 & .078 & .970 & 3.111 & 1.289 & .091 \\
\hline & 2 & .144 & 1.394 & 3.111 & 1.891 & .190 \\
\hline & 5 & .290 & 1.963 & 3.111 & 2.516 & .529 \\
\hline & 10 & .444 & 2.333 & 3.111 & 2.793 & 1.111 \\
\hline & 100 & .938 & 2.980 & 3.111 & 3.077 & 2.824 \\
\hline \multirow[t]{5}{*}{2.00} & 1 & .040 & .504 & 1.556 & .624 & .045 \\
\hline & 2 & .074 & .730 & 1.556 & .919 & .092 \\
\hline & 5 & .155 & 1.033 & 1.556 & 1.238 & .244 \\
\hline & 10 & .247 & 1.222 & 1.556 & 1.384 & .503 \\
\hline & 100 & .641 & 1.509 & 1.556 & 1.537 & 1.395 \\
\hline \multirow[t]{5}{*}{5.00} & 1 & .016 & .206 & .622 & .245 & .018 \\
\hline & 2 & .030 & .300 & .622 & .361 & .036 \\
\hline & 5 & .065 & .424 & .622 & .490 & .093 \\
\hline & 10 & .105 & .499 & .622 & .550 & .188 \\
\hline & 100 & .305 & .606 & .622 & .614 & .553 \\
\hline
\end{tabular}


Table II

\section{Portfolio Weight and Wealth Equivalent Loss Comparisons for the Calibrated Model Where Jumps Occur Every 25 Years on Average}

This table reports portfolio weights for the stochastic volatility model with deterministic jumps in prices and volatility. Also reported are the percentage wealth equivalent losses for an investor who ignores the possibility of event-related jumps. This loss reflects the cost (as a percentage of his wealth) to an investor who assumes that jumps cannot occur, calibrates the model to match historical moments, and follows the portfolio strategy he believes is optimal, but is actually suboptimal in cases where jumps can occur. The average frequency of an event is 25 years. The first column reports the portfolio weights when the jump sizes are both zero (no jumps). The second column reports the portfolio weights and wealth equivalent losses when the stock price jump is -25 percent and the volatility jump is zero (stock jumps only). The third column reports the portfolio weights and wealth equivalent losses for the baseline case where the stock price jump is -25 percent and the volatility jumps to 50 percent. Each scenario is calibrated to match the parameter estimates in Table 1 of Pan (2002).

\begin{tabular}{rrrrrr}
\hline & & & & \multicolumn{2}{c}{$\begin{array}{c}\text { Both Stock and } \\
\text { Volatility Jumps }\end{array}$} \\
$\begin{array}{r}\text { Risk } \\
\text { Aversion } \\
\text { Parameter }\end{array}$ & $\begin{array}{r}\text { Portfolio } \\
\text { Weight }\end{array}$ & $\begin{array}{r}\text { Portfolio } \\
\text { Weight }\end{array}$ & $\begin{array}{r}\text { Equivalent } \\
\text { Loss }\end{array}$ & $\begin{array}{r}\text { Portfolio } \\
\text { Weight }\end{array}$ & $\begin{array}{r}\text { Wealth } \\
\text { Equivalent } \\
\text { Loss }\end{array}$ \\
\hline 50 & 8.106 & 3.914 & 100.0 & 3.865 & 100.0 \\
1.00 & 4.396 & 3.163 & 100.0 & 3.163 & 100.0 \\
2.00 & 2.305 & 1.929 & 3.2 & 2.010 & 2.0 \\
3.00 & 1.564 & 1.356 & 1.3 & 1.432 & .5 \\
4.00 & 1.183 & 1.042 & .7 & 1.107 & .2 \\
5.00 & .952 & .845 & .5 & .901 & .1 \\
\hline
\end{tabular}


Table III

\section{Portfolio Weight and Wealth Equivalent Loss Comparisions for the Calibrated Model Where Jumps Occur Every 100 Years on Average}

This table reports portfolio weights for the stochastic volatility model with deterministic jumps in prices and volatility. Also reported are the percentage wealth equivalent losses for an investor who ignores the possibility of event-related jumps. This loss reflects the cost (as a percentage of his wealth) to an investor who assumes that jumps cannot occur, calibrates the model to match historical moments, and follows the portfolio strategy he believes is optimal, but is actually suboptimal in cases where jumps can occur. The average frequency of an event is 100 years. The first column reports the portfolio weights when the jump sizes are both zero (no jumps). The second column reports the portfolio weights and wealth equivalent losses when the stock price jump is -50 percent and the volatility jump is zero (stock jumps only). The third column reports the portfolio weights and wealth equivalent losses for the baseline case where the stock price jump is -50 percent and the volatility jumps to 70 percent. Each scenario is calibrated to match the parameter estimates in Table 1 of Pan (2002).

\begin{tabular}{rrrrrr}
\hline & & & & \multicolumn{2}{c}{$\begin{array}{c}\text { Both Stock and } \\
\text { Volatility Jumps }\end{array}$} \\
$\begin{array}{r}\text { Risk } \\
\text { Aversion } \\
\text { Parameter }\end{array}$ & $\begin{array}{r}\text { Portfolio } \\
\text { Weight }\end{array}$ & $\begin{array}{r}\text { Portfolio } \\
\text { Weight }\end{array}$ & $\begin{array}{r}\text { Equivalent } \\
\text { Loss }\end{array}$ & $\begin{array}{r}\text { Portfolio } \\
\text { Weight }\end{array}$ & $\begin{array}{r}\text { Wealth } \\
\text { Equivalent } \\
\text { Loss }\end{array}$ \\
\hline 50 & 8.106 & 1.993 & 100.0 & 1.987 & 100.0 \\
1.00 & 4.396 & 1.859 & 100.0 & 1.859 & 100.0 \\
2.00 & 2.305 & 1.395 & 100.0 & 1.481 & 100.0 \\
3.00 & 1.564 & 1.059 & 30.5 & 1.174 & 100.0 \\
4.00 & 1.183 & .844 & 11.2 & .956 & 5.3 \\
5.00 & .952 & .698 & 6.3 & .801 & 2.2 \\
\hline
\end{tabular}




\section{Table IV}

\section{Portfolio Weights for the Calibrated Model for Varying Percentage Price Jumps and Jump Frequencies}

This table reports portfolio weights for the stochastic volatility model with deterministic jump sizes in prices and volatility. Each combination of parameters is calibrated to match the parameter estimates in Table 1 of Pan (2002). The frequency of jumps is expressed in years and equals the reciprocal of the jump intensity. Sets of parameters for which the moments cannot be matched are denoted by a dash.

\begin{tabular}{|c|c|c|c|c|c|c|c|}
\hline \multirow{2}{*}{$\begin{array}{r}\text { Risk } \\
\text { Aversion } \\
\text { Parameter }\end{array}$} & \multirow{2}{*}{$\begin{array}{l}\text { Volatility } \\
\text { Jumps to }\end{array}$} & \multirow{2}{*}{$\begin{array}{r}\text { Frequency } \\
\text { of Jumps }\end{array}$} & \multicolumn{5}{|c|}{ Percentage Price Jump Size } \\
\hline & & & -10 & -20 & -30 & -40 & -50 \\
\hline \multirow[t]{5}{*}{.50} & 25 & 20 & 7.772 & 4.825 & 3.219 & 2.398 & 1.900 \\
\hline & & 30 & 7.871 & 4.903 & 3.272 & 2.444 & 1.945 \\
\hline & & 40 & 7.924 & 4.938 & 3.295 & 2.465 & 1.965 \\
\hline & & 50 & 7.958 & 4.957 & 3.307 & 2.476 & 1.976 \\
\hline & & 100 & 8.029 & 4.987 & 3.326 & 2.493 & 1.993 \\
\hline \multirow[t]{5}{*}{.50} & 50 & 20 & 7.757 & 4.752 & 3.175 & - & - \\
\hline & & 30 & 7.838 & 4.855 & 3.246 & 2.423 & 1.925 \\
\hline & & 40 & 7.892 & 4.906 & 3.278 & 2.452 & 1.953 \\
\hline & & 50 & 7.929 & 4.934 & 3.295 & 2.467 & 1.968 \\
\hline & & 100 & 8.011 & 4.979 & 3.322 & 2.490 & 1.991 \\
\hline \multirow[t]{5}{*}{2.00} & 25 & 20 & 2.287 & 2.091 & 1.687 & 1.325 & 1.066 \\
\hline & & 30 & 2.293 & 2.149 & 1.790 & 1.426 & 1.153 \\
\hline & & 40 & 2.296 & 2.182 & 1.858 & 1.496 & 1.216 \\
\hline & & 50 & 2.298 & 2.204 & 1.908 & 1.549 & 1.264 \\
\hline & & 100 & 2.302 & 2.251 & 2.040 & 1.702 & 1.403 \\
\hline \multirow[t]{5}{*}{2.00} & 50 & 20 & 2.262 & 2.130 & 1.764 & - & - \\
\hline & & 30 & 2.281 & 2.184 & 1.859 & 1.494 & 1.217 \\
\hline & & 40 & 2.288 & 2.211 & 1.920 & 1.557 & 1.270 \\
\hline & & 50 & 2.292 & 2.228 & 1.964 & 1.605 & 1.312 \\
\hline & & 100 & 2.299 & 2.265 & 2.081 & 1.746 & 1.440 \\
\hline \multirow[t]{5}{*}{5.00} & 25 & 20 & .948 & .897 & .776 & .639 & .528 \\
\hline & & 30 & .949 & .913 & .814 & .686 & .572 \\
\hline & & 40 & .950 & .922 & .839 & .719 & .605 \\
\hline & & 50 & .950 & .928 & .855 & .743 & .631 \\
\hline & & 100 & .951 & .939 & .896 & .811 & .708 \\
\hline \multirow[t]{5}{*}{5.00} & 50 & 20 & .928 & .917 & .839 & - & - \\
\hline & & 30 & .939 & .931 & .870 & .762 & .655 \\
\hline & & 40 & .943 & .937 & .885 & .784 & .674 \\
\hline & & 50 & .945 & .940 & .895 & .801 & .693 \\
\hline & & 100 & .949 & .946 & .920 & .852 & .755 \\
\hline
\end{tabular}


Table V

\section{Portfolio Weights for the Calibrated Model for Varying Volatility Jump Sizes and Jump Frequencies}

This table reports portfolio weights for the stochastic volatility model with deterministic jump sizes in prices and volatility. Each combination of parameters is calibrated to match the parameter estimates in Table 1 of Pan (2002). The frequency of jumps is expressed in years and equals the reciprocal of the jump intensity. Set of parameters for which moments cannot be matched are denoted by a dash.

\begin{tabular}{|c|c|c|c|c|c|c|}
\hline \multirow{2}{*}{$\begin{array}{r}\text { Risk } \\
\text { Aversion } \\
\text { Parameter }\end{array}$} & \multirow{2}{*}{$\begin{array}{l}\text { Percentage } \\
\text { Price Jump }\end{array}$} & \multirow{2}{*}{$\begin{array}{l}\text { Frequency } \\
\text { of Jumps }\end{array}$} & \multicolumn{4}{|c|}{ Volatility Jumps to } \\
\hline & & & 20 & 30 & 40 & 50 \\
\hline \multirow[t]{5}{*}{.50} & -25 & 20 & 3.876 & 3.861 & 3.841 & 3.816 \\
\hline & & 30 & 3.932 & 3.924 & 3.912 & 3.896 \\
\hline & & 40 & 3.958 & 3.953 & 3.945 & 3.934 \\
\hline & & 50 & 3.971 & 3.967 & 3.962 & 3.954 \\
\hline & & 100 & 3.992 & 3.991 & 3.989 & 3.986 \\
\hline \multirow[t]{5}{*}{.50} & -50 & 20 & 1.905 & 1.894 & 1.877 & - \\
\hline & & 30 & 1.947 & 1.942 & 1.935 & 1.925 \\
\hline & & 40 & 1.966 & 1.964 & 1.959 & 1.953 \\
\hline & & 50 & 1.977 & 1.975 & 1.972 & 1.968 \\
\hline & & 100 & 1.993 & 1.993 & 1.992 & 1.991 \\
\hline \multirow[t]{5}{*}{2.00} & -25 & 20 & 1.885 & 1.911 & 1.942 & 1.963 \\
\hline & & 30 & 1.976 & 1.996 & 2.022 & 2.044 \\
\hline & & 40 & 2.033 & 2.050 & 2.071 & 2.091 \\
\hline & & 50 & 2.072 & 2.087 & 2.105 & 2.123 \\
\hline & & 100 & 2.167 & 2.177 & 2.188 & 2.199 \\
\hline \multirow[t]{5}{*}{2.00} & -50 & 20 & 1.054 & 1.080 & 1.117 & - \\
\hline & & 30 & 1.146 & 1.163 & 1.188 & 1.217 \\
\hline & & 40 & 1.209 & 1.224 & 1.245 & 1.270 \\
\hline & & 50 & 1.258 & 1.271 & 1.289 & 1.312 \\
\hline & & 100 & 1.399 & 1.409 & 1.423 & 1.440 \\
\hline \multirow[t]{5}{*}{5.00} & -25 & 20 & .833 & .854 & .878 & .887 \\
\hline & & 30 & .864 & .880 & .897 & .090 \\
\hline & & 40 & .882 & .894 & .908 & .918 \\
\hline & & 50 & .894 & .904 & .916 & .924 \\
\hline & & 100 & .920 & .926 & .932 & .937 \\
\hline \multirow[t]{5}{*}{5.00} & -50 & 20 & .515 & .546 & .596 & - \\
\hline & & 30 & .562 & .584 & .616 & .655 \\
\hline & & 40 & .597 & .615 & .641 & .674 \\
\hline & & 50 & .623 & .640 & .663 & .693 \\
\hline & & 100 & .702 & .715 & .733 & .754 \\
\hline
\end{tabular}



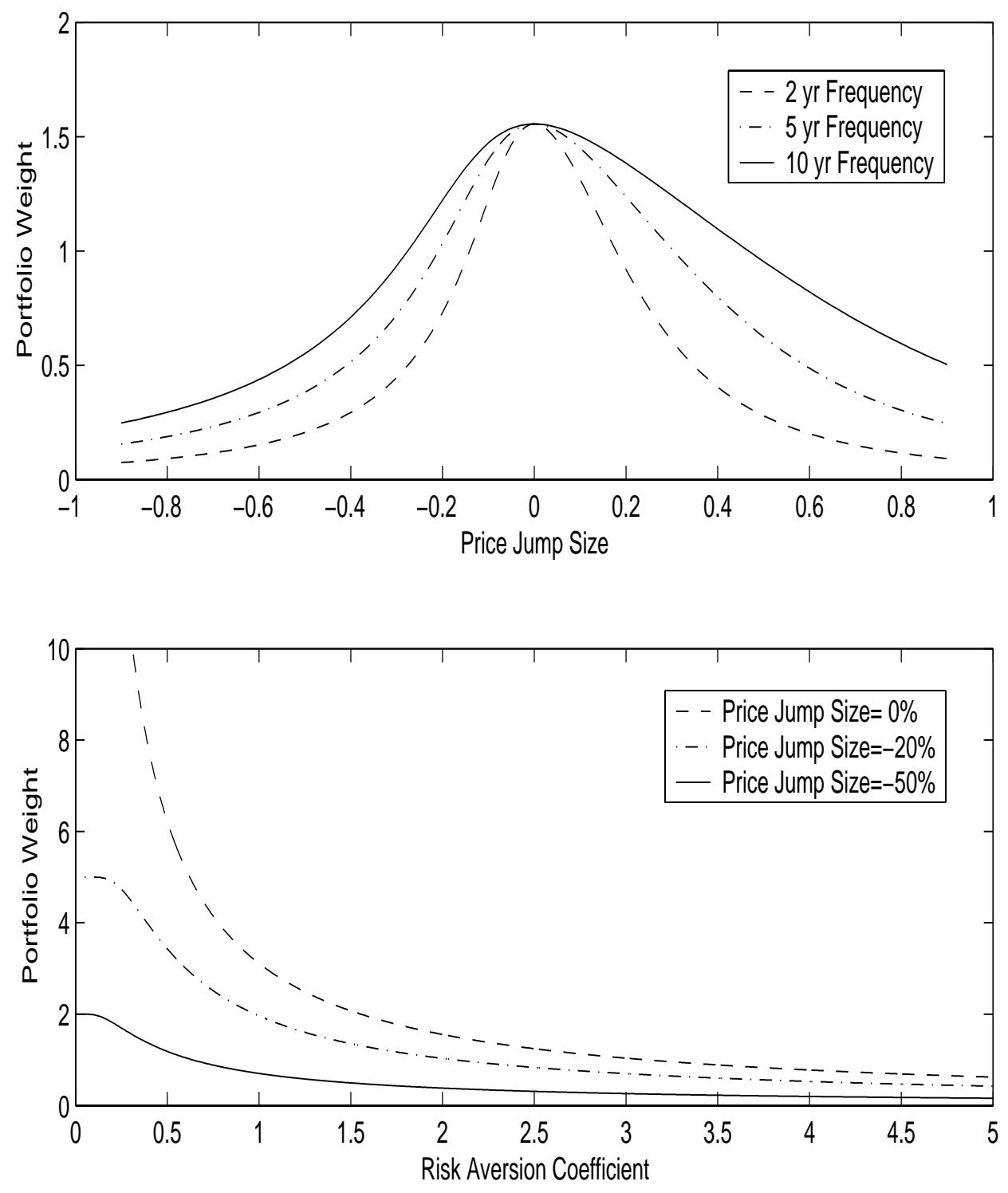
Figure 1. Optimal portfolio weights for the constant-volatility case. The top panel graphs the optimal portfolio weight as a function of the size of the price jump for three different values of the jump frequency. The bottom panel graphs the optimal portfolio weight as a function of the risk aversion coefficient for three different values of the size of the price jump. 

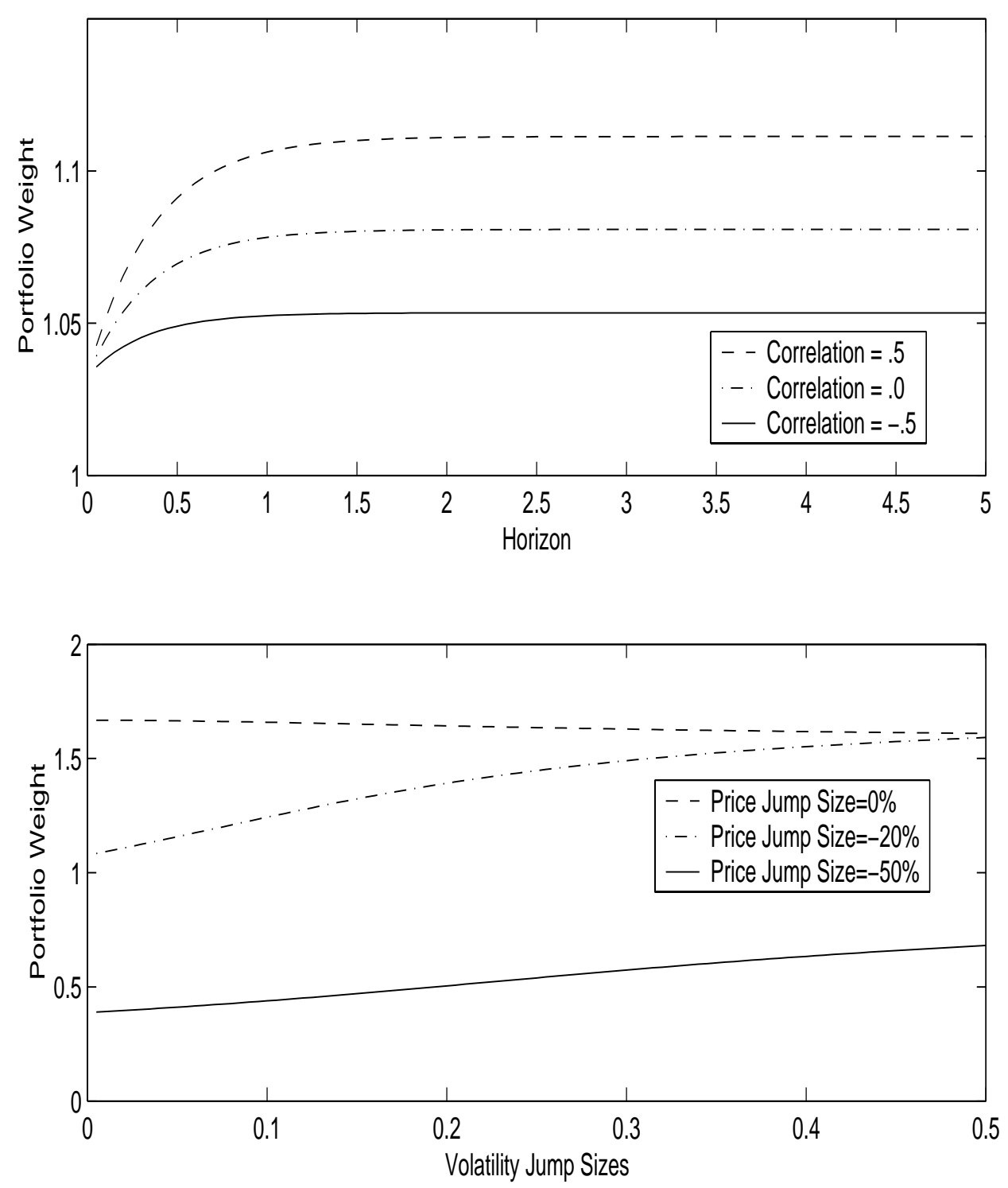
Figure 2. Optimal portfolio weights for the stochastic-volatility case. The top panel graphs the optimal portfolio weight as a function of the investor's horizon measured in years for three different values of the correlation coefficient. The bottom panel graphs the optimal portfolio weight as a function of the size of the volatility jumps for three different values of the size of the price jump. 